\title{
DOS PROBLEMAS DE PROSODIA DEL GRIEGO ANTIGUOO. SEGUINDA PARTE: BARÍTONOS SOBRE SÍLABAS CERRADAS
}

AlejANDRo Abritta
Universidad de Buenos Aires
alejandroabritta88@yahoo.com.ar

El presente artículo es la segunda parte de un estudio sobre dos problemas de la prosodia del griego antiguo vinculados a la relación entre el acento tonal del lenguaje y la estructura silábica de las palabras. En él, luego de una introducción general y un resumen de la primera parte, se analiza el problema de las palabras con acento barítono sobre sílabas cerradas. La principal novedad del análisis es que el corpus de estudio es la poesía homérica, fuente antes desatendida como evidencia del acento.

Prosodia / Griego antiguo / Acento / Sílaba / Homero

\section{TWO PROBLEMS OF ANCIENT GREEK PROSODY PART TWO: BARITONES ON CLOSED SYLLABLES}

This paper is the second part of a study on two problems of Ancient Greek prosody, regarding the relation between the tonal accent of the language and the syllabic structure of words. In it, after a general introduction and a brief sum up of the first part, the problem of words with baritone accent on closed syllable is analyzed. The main novelty of the analysis is that the corpus used is the Homeric poetry, until today unattended as a source of evidence for Greek accent.

Prosody / Ancient Greek / Accent / Syllable / Homer 


\subsection{Introducción}

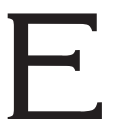

I presente trabajo intenta encarar, como indica su título, dos problemas de la prosodia del griego antiguo ${ }^{1}$. Ya se ha analizado el primero en la primera parte, esta segunda se dedicará al análisis del otro. Ambos conciernen a la relación entre los distintos tipos de sílabas cerradas en la lengua y la contonación que constituye, de acuerdo a AlLEN (1973 y 1987), al que se seguirá aquí, su acento. Trabajos anteriores (en particular, los de Devine y Stephens (especialmente Devine y Stephens, 1994) han encarado problemas similares a partir de un estudio de los textos con notación musical preservados, sobre la premisa metodológica de que éstos son la única fuente directa con la que contamos para un estudio del acento griego. Sin embargo, en este trabajo, y siguiendo las investigaciones de Nagy (1996: 129-132, 2000 y 2010) David (2006) se utilizará un criterio distinto, en la medida en que se aceptará que el acento tonal de la lengua griega cumplía un rol en la poesía griega. Por ello, el corpus que se utilizará son los poemas homéricos ${ }^{2}$, y el análisis no dependerá de una comparación entre los acentos gráficos y la notación musical sino de un estudio de la distribución de esos acentos.

En la próxima sección dentro de esta introducción realizaré un breve resumen de algunos conceptos teóricos ya mencionados y utilizados en la primera parte. Los lectores que no estén familiarizados con ellos deberían revisar la sección 1 de ese artículo. El resto de éste se dedica a analizar el segundo problema que se encarará, a partir del mismo esquema que se utilizó anteriormente: formulación, metodología, prueba y conclusiones. Recomiendo enfáticamente al lector que no esté interesado en seguir en detalle los análisis estadísticos que se realizarán pasar directamente de la formulación del problema (sec. 3.1) a las conclusiones (sec. 3.4), donde se resumen los resultados alcanzados.

1 A partir de este punto, "griego" quiere decir siempre "griego antiguo".

2 Más específicamente, una selección de 2484 versos de los primeros doce cantos de la Ilíada y los veinticuatro de la Odisea (pero con una cantidad similar de versos de cada poema). La constitución exacta del corpus, las ediciones utilizadas, los datos de este estudio y aquellos sobre los que estos se basan pueden hallarse en https://greekmps.wordpress.com. 


\subsection{Resumen de la primera parte \\ 0.2.1. Conceptos teóricos}

Una serie de oposiciones prosódicas constituye el conjunto de los principales conceptos teóricos utilizados en este trabajo:

Sílabas largas/breves - Vocales largas/breves: Debe tenerse en cuenta que estas dos oposiciones son independientes la una de la otra, a pesar de estar fuertemente vinculadas. La categoría de las sílabas cerradas, que es central en este estudio, demuestra esto claramente, en la medida en que una sílaba cerrada es una sílaba larga con vocal breve ${ }^{3}$.

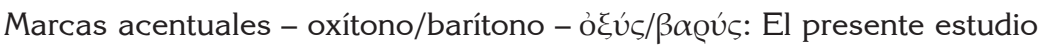
utiliza la clasificación tradicional (oxítonas, paroxítonas, proparoxítonas, perispómenas y properispómenas, siempre en femenino) exclusivamente para referirse a las marcas acentuales de las palabras, no a su acento. A su vez, utiliza "oxítono" y "barítono" (en masculino) para referirse a las partes del acento griego (respectivamente, el tono ascendente y el tono descendente $)^{4}$. La clasificación que identifica el tipo de acento de las palabras

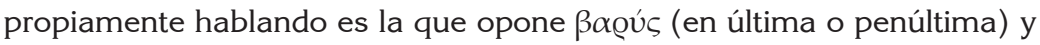
ò Łús (en última, penúltima o antepenúltima), formulada en DAviD (2006).

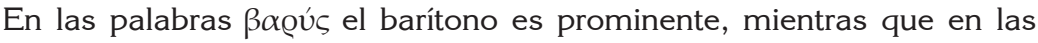
palabras ỏ Ł́v́c lo es el oxítono ${ }^{5}$. Qué parte de la contonación es prominente depende de en qué tipo de sílaba cae cada parte de la contonación.

3 Estrictamente hablando, "cerrada" se refiere a una característica de cualquier sílaba, no sólo a aquellas que tienen una vocal breve. Las sílabas superlargas son sílabas cerradas con vocal larga. Dado, sin embargo, que aquí no se considerará la diferencia entre largas y superlargas, y que sería tedioso indicar a cada paso "cerrada con vocal breve", en el presente trabajo "sílaba cerrada" quiere decir siempre "sílaba cerrada con vocal breve".

Estrictamente hablando, "cerrada" se refiere a una característica de cualquier sílaba, no sólo a aquellas que tienen una vocal breve. Las sílabas superlargas son sílabas cerradas con vocal larga. Dado, sin embargo, que aquí no se considerará la diferencia entre largas y superlargas, y que sería tedioso indicar a cada paso "cerrada con vocal breve", en el presente trabajo "sílaba cerrada" quiere decir siempre "sílaba cerrada con vocal breve". Estrictamente hablando, "cerrada" se refiere a una característica de cualquier sílaba, no sólo a aquellas que tienen una vocal breve. Las sílabas superlargas son sílabas cerradas con vocal larga. Dado, sin embargo, que aquí no se considerará la diferencia entre largas y superlargas, y que sería tedioso indicar a cada paso "cerrada con vocal breve", en el presente trabajo "sílaba cerrada" quiere decir siempre "sílaba cerrada con vocal breve". 


\subsubsection{Conclusiones de la primera parte}

Un análisis de la distribución de las palabras de final trocaico con acento agudo en penúltima demostró que las sílabas cerradas por resonante con acento agudo en penúltima (como en ع́ $\gamma \chi 0 \varsigma$ ) se asimilaban a los $\beta \alpha \varrho u ́ \varsigma$ en

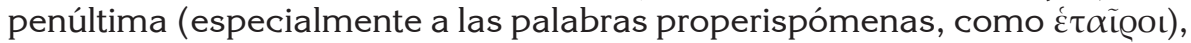
mientras que las sílabas cerradas por oclusiva con acento agudo (como en

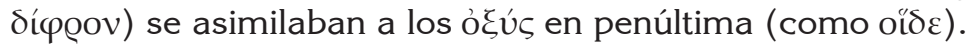

\section{Barítono sobre sílabas cerradas}

\subsection{Formulación del problema}

El acento barítono sigue siempre al oxítono, a menos que la mora que carga con el segundo sea la última de la palabra. De acuerdo con DAVID

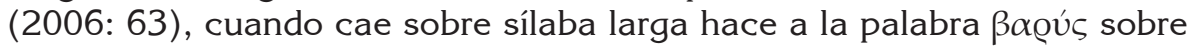
esa sílaba larga. Cuando cae sobre vocal larga, no hay mayor inconveniente, porque esta vocal tiene dos moras sobre las que puede completarse la contonación; pero cuando cae sobre sílaba cerrada sólo hay una mora vocálica para que esto suceda. El problema que se intentará resolver a continuación es si se percibía una diferencia entre barítonos sobre

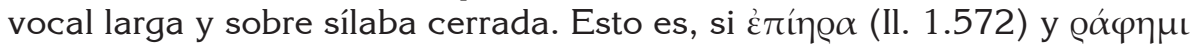

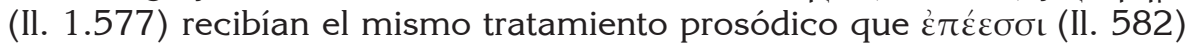

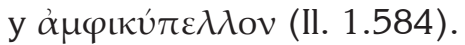

Como en el caso de las sílabas cerradas con agudo, pero en este caso a priori, dado que los autores consultados no discuten el problema, hay tres posibilidades:

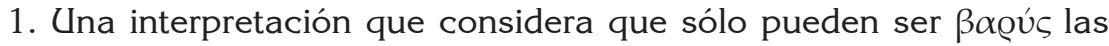
palabras que tienen barítono sobre sílaba larga.

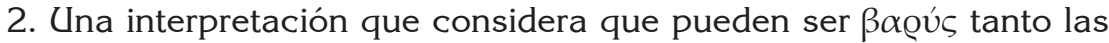
palabras con barítono sobre vocal larga como las palabras con barítono sobre sílaba cerrada, pero sólo sobre sílaba cerrada con resonante (en analogía al caso de los agudos sobre sílaba cerrada).

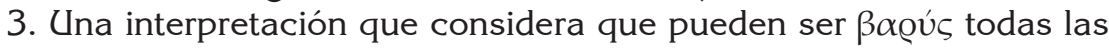
palabras con barítono sobre sílaba cerrada.

\subsection{Metodología}

A priori la metodología utilizada en el problema anterior podría funcionar para encarar éste. Sin embargo, un análisis preliminar de los datos indica que no hay una ubicación del metro donde se perciba claramente una preferencia o una evasión de cierto tipo de acento que permita discriminar entre las diferentes interpretaciones prosódicas de las formas en 
cuestión. Se necesita utilizar una aproximación menos localizada pero más abarcativa para solucionar la dificultad que se plantea.

El primer paso es estudiar el comportamiento de las formas que, independientemente del contexto en el que aparecen, tienen la estructura

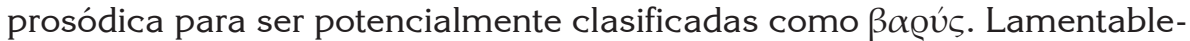
mente, los únicos tipos de palabra que se adecuan a esta exigencia son, nuevamente, los de final trocaico, específicamente las palabras trisilábicas y mayores de final trocaico. Dentro de éstas hay dos grupos claramente diferentes en su distribución en el metro: el de palabras de final anfibráqueo $(\mathrm{u}-\mathrm{u})^{6}$ y el de palabras de final palimbáquico $(--\mathrm{u})^{7}$. (Interesantemente, son los grupos respectivamente mejor y peor representados en el corpus analizado.) Si se hallara que en todos los casos, sea cual sea el tipo de sílaba en la penúltima, se comportan de manera similar, entonces podría concluirse tentativamente que la interpretación que las considera universalmente $\beta \alpha$ @ús en penúltima es correcta.

Un segundo paso es analizar la distribución de las formas que sólo en sandhi ${ }^{8}$ responden a los parámetros de la prueba. Este grupo se divide a su vez en dos: aquel en donde no cambia el valor de la sílaba con el barítono y aquel en donde lo hace. En el primer grupo entran las formas trisilábicas y mayores con sílaba final cerrada y final espondaico ${ }^{9}$. Este mismo tipo de de estructura silábica entra en el segundo grupo, pero en este caso las formas en consideración no son las proparoxítonas (donde la sílaba con el barítono es larga en la forma aislada) sino las paroxítonas (donde la sílaba con el barítono es breve en la forma aislada). Una palabra como $\kappa \alpha i ́ \omega \mu \varepsilon v$ entra en el primer grupo porque independientemente de su con-

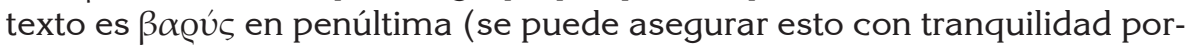
que la vocal es larga), pero una palabra como * $\kappa \alpha \iota \omega \mu \varepsilon v$ entraría en el se-

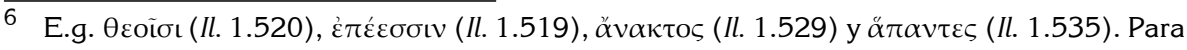
todas las formas que se mencionarán daré ejemplos del corpus seleccionado en este orden (vocal larga, cerrada por sibilante, por oclusiva y por resonante).

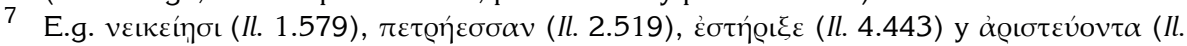
7.90). En el análisis de estas formas, por motivos que están relacionados con la complejidad del sistema de análisis de los datos, consideraré sólo aquellas con una vocal larga o diptongo en la antepenúltima (casi un $75 \%$ del total de estas formas).

"Sandhi" es el término sánscrito, hoy adoptado en lingüística, para referirse a los cambios fonéticos que las palabras sufren en determinados contextos fonológicos, es decir, en su contacto con otras.

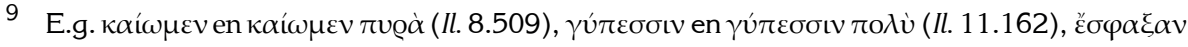

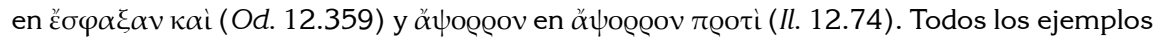
son molosos (-- -) con final cerrada por líquida (de hecho, nasal). Dada la cantidad absoluta de este grupo, no distinguiré en él entre molosos ( $u--)$ y báqueos ( $u--)$. Naturalmente, qué tipo de sílaba cerrada final tengan es intrascendente a los fines del análisis. Nótese también que estas palabras son las mismas que, si su sílaba final no estuviera cerrada, estarían en el primer grupo de análisis (anfibráqueos y palimbáqueos). 
gundo si en un contexto específico su sílaba final apareciera cerrada por la consonante inicial de la palabra siguiente. Lo mismo puede decirse de las palabras de final yámbico ${ }^{10}$. Este grupo de formas que sólo tienen barítono en sílaba larga cuando su sílaba final está cerrada es, por así decirlo, el más sospechoso de todos, dado que implican una modificación sustancial de la forma lingüística base.

En todos los casos se compararán porcentajes de distribución de las palabras donde el barítono cae sobre vocal larga con aquellos donde caería en sílaba cerrada con oclusiva y en sílaba cerrada con resonante ${ }^{11}$. Si se encontraran en cualquiera de los casos, pero sobre todo en los primeros, diferencias significativas entre los diferentes tipos de sílaba, podría concluirse que la estructura silábica afecta la percepción de la naturaleza acentual de la palabra.

\subsection{Pruebas}

3.3.1. Formas de final trocaico

3.3.1.1. Anfibráqueos

Como en la primera parte, para no generar distorsiones, el análisis excluye enclíticos y proclíticos (como $\varepsilon \sigma \tau \iota$ y $\alpha \lambda \lambda \alpha \alpha ́$ ), así como palabras métricas (o grupos apositivos) constituidos por una ortotónica y un enclítico, como

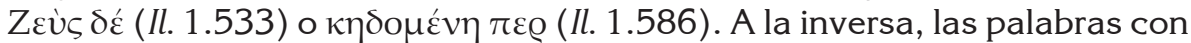
final elidido han sido incluidas, provisto que la forma de hecho en la que

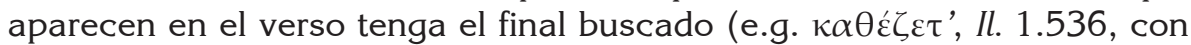
final anfibráqueo). Lo mismo vale para palabras con vocal final abreviada, como 'Họ en 'Họ ö $\tau$ ' (Il. 1.519). En todos los análisis de este trabajo he excluido las palabras con grave final ${ }^{12}$.

La presentación de los resultados resulta difícil, dado que las tablas de análisis contienen muchos datos que podrían resultar útiles. Para simplificar, en la tabla 3.3.1.1 se presenta el porcentaje por pie que representan las formas proparoxítonas con diferentes estructuras silábicas. En este caso (cf. sec. 2.4.3, n. 41 en la primera parte) las properispómenas

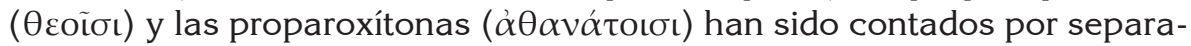
do en el caso de las palabras con vocal larga en penúltima, porque el objetivo es contrastar el comportamiento específico de los barítonos en síla-

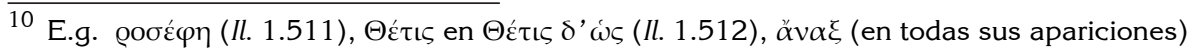

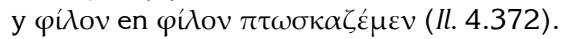

11 Como en la primera parte (n. 12), para simplificar la exposición utilizaré "oclusivas" para referirme tanto a la oclusivas propiamente dichas como a la sibilante ó́ $\gamma \mu \alpha$. La terminología es, evidentemente, inapropiada. Sin embargo los resultados obtenidos hasta este punto sugieren que analizar los dos grupos por separado no proporciona ninguna ventaja. En todo caso, en el blog mencionado en la nota 2 puede hallarse el análisis desagregado.

12 Pero pueden hallarse las pruebas con las graves en https://greekmps.wordpress.com. 
ba larga. Dados los resultados de la sección 2 en la primera parte de este trabajo, oponer el porcentaje de la suma de los circunflejos y los barítonos post-agudo en el caso de las palabras con vocal larga al porcentaje de las proparoxítonas en las palabras con sílaba cerrada distorsionaría los resul-

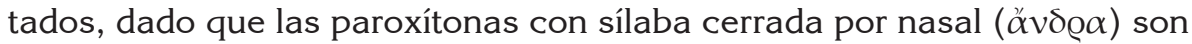

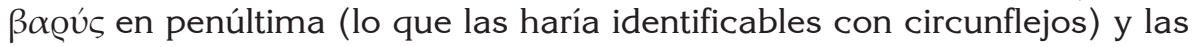
paroxítonas en sílaba cerrada por oclusiva (ỏ $\varphi \varrho \alpha)$ son ỏ $\xi u ́ s$ en penúltima (lo que las haría distintas de los circunflejos).

\begin{tabular}{|l|r|r|r|r|r|}
\hline \% Col. Pro. Fin. Anf. & \multicolumn{2}{c}{ 2 ${ }^{\circ}$ pie } & \multicolumn{2}{c}{ 3 pie } & \multicolumn{2}{c|}{ 5 $^{\circ}$ pie } & pie & Total \\
\hline Penúlt. vocal larga & $70,83 \%(24)$ & $76,15 \%(327)$ & $75 \%(112)$ & $74,21 \%(535)$ & $74,85 \%(998)$ \\
\hline Penúlt. oclusiva & $80 \%(10)$ & $88,64 \%(88)$ & $95,65 \%(23)$ & $93,04 \%(158)$ & $91,40 \%(279)$ \\
\hline Penúlt. resonante & $100 \%(6)$ & $77,03 \%(74)$ & $88,57 \%(35)$ & $79,74 \%(153)$ & $80,60 \%(268)$ \\
\hline
\end{tabular}

Tabla 3.3.1.1. Porcentajes por ubicación de las palabras anfibráquicas proparoxítonas con diferentes estructuras silábicas en diferentes ubicaciones del metro en Homero. Entre paréntesis se agrega el total de palabras (esto es, la suma de todos los tipos acentuales) de cada estructura en cada ubicación.

Se identifica la ubicación de la sílaba final de la palabra a partir del pie en donde cae, ya que las palabras de final trocaico sólo pueden aparecer con la penúltima en la tesis de un pie dactílico. Como en las tablas de la sección 2 en la primera parte, se ha excluido el cuarto pie, en la medida en que allí el puente de Hermann disminuye significativamente la aparición de palabras de final trocaico y, dados los resultados de CANTILENA (1995), se ha incluido el segundo. El primero ha quedado fuera del análisis por la sencilla razón de que los trisílabos de final trocaico deberían empezar antes que el verso, lo que es imposible (i.e., que los trisílabos de esta configuración no entran en el primer pie). En sí mismos, estos resultados, aunque compatibles con la idea de que todas las proparoxítonas de final trocaico, independientemente de su estructura silábica, son $\beta \alpha \rho v ́ \varsigma$ en penúltima, no permiten obtener conclusiones definitivas. Es interesante notar que las palabras con oclusiva son las que mayor cantidad (relativa) de proparoxítonas usan en cada posición, en particular porque son el único tipo que no tiene otra alternativa para formar $\beta \alpha \rho v ́$ (las palabras con vocal larga pueden serlo siendo properispómenas y las palabras con resonante pueden serlo siendo paroxítonas). Más allá de eso, es imposible saber si estos números son el resultado de una distribución deliberada o el simple subproducto del hecho de que el lenguaje no provee suficientes formas alternativas a las proparoxítonas en estas estructuras ${ }^{13}$.

13 Estrictamente hablando, esta imposibilidad podría subsanarse con un estudio integral de los rasgos prosódicos y acentuales del léxico homérico, que contemple además la distribución 


\subsubsection{Palimbáqueos}

Se aplican los mismos criterios de análisis que para los anfibráqueos. La tabla 3.3.1.2. muestra los porcentajes por ubicación de estas formas.

\begin{tabular}{|c|c|c|c|c|c|}
\hline \% Col. Pro. Fin. Pal. & $2^{\circ}$ pie & $3^{\circ}$ p pie & $5^{\circ}$ pie & $6^{\circ}$ pie & Total \\
\hline Penúlt. vocal larga & $61,54 \%(13)$ & $70,10 \%(97)$ & $68,00 \%(75)$ & $63,33 \%(30)$ & $67,91 \%(215)$ \\
\hline Penúlt. oclusiva & $100 \%(2)$ & $100 \%(36)$ & $100 \%(24)$ & $100 \%(10)$ & $100 \%(72)$ \\
\hline Penúlt. resonante & $100 \%(5)$ & $88,24 \%(34)$ & $73,68 \%(19)$ & $83,33 \%(24)$ & $84,15 \%(82)$ \\
\hline
\end{tabular}

Tabla 3.3.1.2. Porcentajes por ubicación de las palabras palimbáquicas proparoxítonas con diferentes estructuras silábicas en diferentes ubicaciones del metro en Homero. Entre paréntesis se agrega el total de palabras (esto es, la suma de todos los tipos acentuales) de cada estructura en cada ubicación.

Una vez más, los resultados son compatibles con la interpretación que

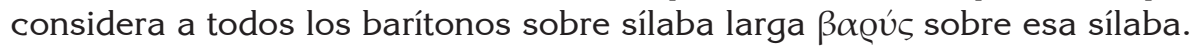
Nuevamente, las palabras con oclusiva muestran una preferencia extremadamente (muy extremadamente, en este caso) marcada por este tipo acentual en todas las ubicaciones, mientras que, llamativamente, las palabras con vocal larga tienen números relativamente bajos ${ }^{14}$. Debe señalarse, sin embargo, que no puede asegurarse que estos números sean algo más que el subproducto de la distribución de tipos acentuales en estas formas en el lenguaje.

\subsubsection{Conclusiones}

Los análisis realizados, junto con los disponibles en https://greekmps.wordpress.com sugieren que es probable que todos los barítonos sobre todas las sílabas largas, independientemente de su estructura silábica, hicieran a la palabra ßa@ús sobre la sílaba larga. No se observaron diferencias significativas en la distribución de palabras proparoxítona con vocal larga en

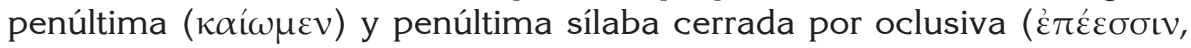
$\ddot{\alpha} v \alpha \kappa \tau \varepsilon \varsigma$ ) o por resonante ( $\alpha \check{\pi} \alpha \nu \tau \varepsilon \varsigma$ ), esto es, en palabras con barítono en

métrica de cada lexema. Sin embargo, aun completando esta titánica tarea (en forma que permita un análisis estadístico comprehensivo de los datos), todavía seguiríamos sin saber si en el lenguaje que Homero conocía había formas que fueron excluidas porque sus rasgos prosódicos no eran compatibles con el sonido que se pretendía para la composición.

14 Esto es producto fundamentalmente de la exclusión de los circunflejos, dado que, en total, solamente hay 6 palabras (todas ỏsús en penúltima; no hay ningún palimbáqueo oxítono)

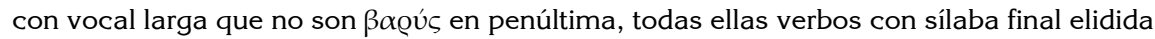
( 
penúltima sílaba larga. Más aún, el hecho de que las palabras con oclusiva en penúltima, cuya estructura silábica no admite otros tipos acentuales ßa@ús más que las proparoxítonas, sean aquellas que mayor preferencia exhiben por esta posición del acento refuerza esta idea. La validez de estos resultados se limita a las palabras cuya sílaba larga en penúltima es también larga en el lenguaje, independientemente del contexto lingüístico. Las pruebas en las siguientes secciones intentarán estudiar si éste es el caso también para otros tipos.

\subsubsection{Penúltimas sílabas en final espondaico}

Esta categoría es intermedia entre la analizada en el apartado anterior, cuya estructura silábica es idéntica a la de las formas aisladas en todas las posiciones, y las que serán analizadas en el siguiente, donde cambia la cantidad de la sílaba en la que cae el barítono. En este grupo la sílaba acentuada tiene la misma cantidad que la forma aislada, pero la sílaba final de la palabra está cerrada en el contexto del verso (cf. los ejemplos en la n. 9).

Las cantidades absolutas de estas formas son lamentablemente demasiado bajas como para realizar un análisis concluyente. Solamente hay tres ubicaciones en el metro con suficiente cantidad de casos en todas las estructuras silábicas como para al menos tomarlas en cuenta. Las tablas 3.3.2.1, 3.3.2.2 y 3.3.2.3 muestran la distribución de tipos de acento en cada una de esas ubicaciones, junto con los porcentajes del total por columna de cada celda. En el título de las columnas, "T" indica "tesis", y cada número el pie en donde termina la palabra.

\begin{tabular}{|l|r|r|r|r|}
\hline Fin. Esp. (Penúl. Larga) & \multicolumn{1}{c}{ T2 } & \multicolumn{1}{c}{ T3 } & \multicolumn{1}{c|}{ Total } \\
\hline Oxítonas & $1(1,54 \%)$ & $1(0,99 \%)$ & $2(3,03 \%)$ & $4(1,72 \%)$ \\
\hline Paroxítonas & $1(1,54 \%)$ & $1(0,99 \%)$ & $4(6,06 \%)$ & $6(2,59 \%)$ \\
\hline Properispómenas & $40(61,54 \%)$ & $39(38,61 \%)$ & $26(39,39 \%)$ & $105(4,31 \%)$ \\
\hline Proparoxítonas & $23(35,38 \%)$ & $60(59,41 \%)$ & $34(51,52 \%)$ & $117(50,43 \%)$ \\
\hline Total & 65 & 101 & 66 & 232 \\
\hline
\end{tabular}

Tabla 3.3.2.1. Cantidades por ubicación de palabras de final espondaico, última sílaba cerrada y penúltima con vocal larga con diferentes tipos de acento en Homero. Entre paréntesis se agrega el porcentaje por columna. 


\begin{tabular}{|l|r|r|r|r|}
\hline Fin. Esp. (Penúl. Oclu.) & \multicolumn{1}{r|}{ T2 } & \multicolumn{2}{r|}{ T3 } & Total \\
\hline Oxítonas & $0(0 \%)$ & $1(14,29 \%)$ & $0(0 \%)$ & $3(5,88 \%)$ \\
\hline Paroxítonas & $3(23,08 \%)$ & $6(57,14 \%)$ & $3(27,27 \%)$ & $28(54,90 \%)$ \\
\hline Proparoxítonas & $10(76,92 \%)$ & $2(28,57 \%)$ & $8(72,73 \%)$ & $20(39,22 \%)$ \\
\hline Total & 13 & 10 & 11 & 51 \\
\hline
\end{tabular}

Tabla 3.3.2.2. Cantidades por ubicación de palabras de final espondaico, última sílaba cerrada y penúltima con sílaba cerrada por oclusiva con diferentes tipos de acento en Homero. Entre paréntesis se agrega el porcentaje por columna.

\begin{tabular}{|l|r|r|r|r|}
\hline Fin. Esp. (Penúl. Reso.) & \multicolumn{1}{c}{ T2 } & \multicolumn{1}{c}{ T3 } & \multicolumn{1}{c|}{ T5 } \\
\hline Oxítonas & $0(0 \%)$ & $0(0 \%)$ & $0(0 \%)$ & $0(0 \%)$ \\
\hline Paroxítonas & $6(31,58 \%)$ & $6(42,86 \%)$ & $0(0 \%)$ & $32(52,26 \%)$ \\
\hline Proparoxítonas & $13(68,42 \%)$ & $8(57,14 \%)$ & $8(100 \%)$ & $29(47,54 \%)$ \\
\hline Total & 19 & 14 & 8 & 82 \\
\hline
\end{tabular}

Tabla 3.3.2.3. Cantidades por ubicación de palabras de final espondaico, última sílaba cerrada y penúltima con sílaba cerrada por resonante con diferentes tipos de acento en Homero. Entre paréntesis se agrega el porcentaje por columna.

Los resultados tienen un cierto grado de incertidumbre en la medida en que todavía no se ha analizado cómo deben interpretarse las palabras de forma paroxítona en estas estructuras. Por lo demás, las cantidades son sin duda demasiado pequeñas como para alcanzar conclusiones certeras. No parece haber evidencia aquí de una preferencia o una evasión peculiar por ninguna forma; únicamente merecen mencionarse las paroxítonas

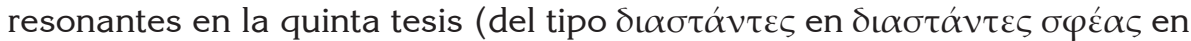
la tercera tesis de Il. 12.86), completamente inexistentes (aunque son una forma poco representada en las otras tablas también), pero este tipo no afecta las conclusiones sobre la naturaleza de las proparoxítonas.

A diferencia de lo que se observó en la sección anterior, las formas oclusivas no parecen aquí exacerbar el uso de barítonos sobre sílabas cerradas contra otros tipos acentuales, excepto quizás en la segunda tesis

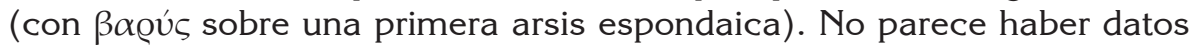
suficientes en estas tablas para reforzar la hipótesis que resultó más favorecida en la sección anterior, pero tampoco para rechazarla. Estos espondeos (como los que serán analizados a continuación), por lo demás, resultan formas poco útiles a los fines del análisis, dado que sus posibles tipos acentuales son muchos y sus cantidades absolutas son relativamente pocas. 


\subsubsection{Silabas cerradas finales}

3.3.3.1. Sílabas finales de espondeo

Aunque una vez más se analizan espondeos, en este caso se centrará la atención en las sílabas finales, y en particular en el contraste entre formas con sílaba final con vocal larga y formas con sílaba final cerrada. La estructura de la penúltima sílaba resulta ahora indiferente para el análisis. En la tabla 3.3.3.1 pueden verse los porcentajes de palabras paroxítonas sobre el total de cada ubicación con más de diez palabras de la estructura en cuestión (los totales se han colocado entre paréntesis) para cada una de las estructuras analizadas. ${ }^{15} \mathrm{He}$ excluido del total de las palabras con vocal larga los casos de las properispómenas con diptongo cerrado en sílaba final (como $\pi \varrho \tilde{\eta} \xi \alpha \iota$ en $\pi \varrho \tilde{\eta} \xi \alpha \iota \delta^{\prime} \varepsilon^{\prime} \mu \pi \eta \varsigma$ de Il. 1.562), porque éstas son problemáticas en sí mismas. "A" indica "arsis" (naturalmente larga) y, como antes, "T" quiere decir tesis; los números indican el pie.

\begin{tabular}{|l|r|r|r|r|r|}
\hline $\begin{array}{l}\text { \% Col. Par. } \\
\text { Fin. Esp. }\end{array}$ & \multicolumn{2}{c}{ A1 } & \multicolumn{2}{c}{ T2 } & \multicolumn{2}{c|}{ T3 } & \multicolumn{1}{c|}{ T5 } & \multicolumn{1}{c|}{ Total } \\
\hline Última vocal larga & $70,83 \%(240)$ & $78 \%(150)$ & $81,69 \%(284)$ & $70,91 \%(110)$ & $66,20 \%(2343)$ \\
\hline Última oclusiva & $36 \%(50)$ & $17,24 \%(58)$ & $26,83 \%(82)$ & $13,51 \%(37)$ & $26,62 \%(278)$ \\
\hline Última resonante & $43,14 \%(51)$ & $6,38 \%(47)$ & $7,04 \%(71)$ & $6,12 \%(49)$ & $17,08 \%(240)$ \\
\hline
\end{tabular}

Tabla 3.3.3.1. Porcentajes por ubicación de las palabras espondaicas paroxítonas con diferentes estructuras silábicas en diferentes ubicaciones del metro en Homero. Entre paréntesis se agrega el total de palabras (esto es, la suma de todos los tipos acentuales) de cada estructura en cada ubicación.

Los datos muestran una predecible tendencia en el caso de las palabras con vocal larga en penúltima a ser paroxítonas, frente a una tendencia a no serlo en el caso de las palabras con última sílaba cerrada. De hecho, contra lo que se hubiera esperado dados los resultados de la primera parte de este trabajo, esto se exacerba en el caso de las palabras con resonante, donde (excepto en el primer pie), prácticamente hay una evasión de estas formas. Esto sugiere, en principio, que se percibía una diferencia clara entre las paroxítonas con final espondaico puro, por así decirlo, y aquellas que sólo tienen final espondaico en sandhi.

Dicho esto, un análisis más comprehensivo de la distribución de los tipos de acento parece necesario en este caso, en la medida en que resulta extraño que las expectativas con respecto a la distribución de acentos en-

15 Estrictamente hablando, más de diez palabras excluyendo las properispómenas, que en este análisis resultan un tanto disruptivas. 
tre los distintos tipos de sílabas cerradas se inviertan. Si bien es esperable que en la poesía no se alteren sustancialmente los tipos acentuales de las palabras, no parece razonable que se evadan las formas con resonantes más que las formas con oclusiva, dados los resultados de la primera parte de este trabajo. Para entender qué está sucediendo, las tablas 3.3.3.2, 3.3.3.3 y 3.3.3.4 muestran las posiciones de los acentos para cada estructura silábica en las posiciones analizadas en la tabla anterior.

\begin{tabular}{|l|r|r|r|r|r|}
\hline Fin. Esp. (Últ. Larga) & \multicolumn{1}{c}{ A1 } & \multicolumn{2}{c}{ T2 } & \multicolumn{2}{c|}{ T3 } \\
\hline Oxítonas & 11 & 6 & 14 & 9 & 40 \\
\hline Paroxítonas & 170 & 117 & 232 & 78 & 403 \\
\hline Perispómenas & 59 & 27 & 38 & 23 & 341 \\
\hline Total & 240 & 150 & 284 & 110 & 784 \\
\hline
\end{tabular}

Tabla 3.3.3.2. Cantidades por ubicación de palabras de final espondaico y última sílaba con vocal larga con diferentes tipos de acento en Homero.

\begin{tabular}{|l|r|r|r|r|r|}
\hline Fin. Esp. (Últ. Oc.) & \multicolumn{2}{c}{ A1 } & \multicolumn{2}{c}{ T2 } & \multicolumn{2}{c|}{ T5 } \\
\hline Oxítonas & 2 & 2 & 7 & 2 & 14 \\
\hline Paroxítonas & 18 & 10 & 22 & 5 & 74 \\
\hline Proparoxítonas & - & 22 & 19 & 15 & 61 \\
\hline Properispómenas & 30 & 24 & 34 & 15 & 103 \\
\hline Total & 20 & 34 & 48 & 22 & 149 \\
\hline
\end{tabular}

Tabla 3.3.3.3. Cantidades por ubicación de palabras de final espondaico y última sílaba cerrada por oclusiva con diferentes tipos de acento en Homero.

\begin{tabular}{|l|r|r|r|r|r|}
\hline Fin. Esp. (Últ. Re.) & \multicolumn{2}{c}{ A1 } & \multicolumn{2}{c}{ T2 } & \multicolumn{2}{c|}{ T5 } \\
\hline Oxítonas & 1 & 4 & 6 & 0 & 12 \\
\hline Paroxítonas & 22 & 3 & 5 & 3 & 41 \\
\hline Proparoxítonas & - & 24 & 34 & 27 & 86 \\
\hline Properispómenas & 28 & 16 & 26 & 19 & 89 \\
\hline Total & 23 & 31 & 45 & 30 & 139 \\
\hline
\end{tabular}

Tabla 3.3.3.4. Cantidades por ubicación de palabras de final espondaico y última sílaba cerrada por resonante con diferentes tipos de acento en Homero. 
Ciertas tendencias se hacen en estas tablas más claras que en la anterior. Primero, la forma lingüística prevalece muy claramente en las palabras que terminan en sílaba cerrada: las proparoxítonas y las properispómenas aparecen con enorme libertad y en gran mayoría en todas las posiciones. Esto, por otro lado, explica los números relativamente bajos de paroxítonas en la tabla 3.3.3.1. La interferencia de los tipos de acento propios de las palabras en su forma aislada hace difícil obtener resultados del todo confiables sobre las palabras en donde el tipo acentual cambia; en otras palabras, hay pocas paroxítonas simplemente porque el lenguaje ofrece pocas paroxítonas.

Un dato, sin embargo, sugiere que las tendencias de estas tablas, opuestas a las observadas en la sección 3.3.1, deben ser tomadas con cuidado. Mientras que en el caso de las palabras con final resonante las cantidades de oxítonas (como ỏ $\rho \chi \eta \sigma \tau \hat{v} v$ en $o j \chi \chi \eta \tau \tau \dot{v} v \tau \varepsilon$ de Od. 1.121) y paroxítonas (como $\mu \dot{\varepsilon} \sigma \sigma o v$ en $\mu \dot{\varepsilon} \sigma \sigma o v \pi \varepsilon \delta i o v$ de Il. 11.172) son similares en la segunda y la tercera tesis, donde se esperaría una mayor cantidad de $\beta \alpha \rho v \zeta,{ }^{16}$ en el caso de las palabras con oclusiva hay una cantidad mucho

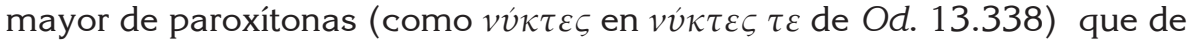
oxítonas (como $\pi \varepsilon \zeta o ́ \varsigma$ en $\pi \varepsilon \zeta o ́ \varsigma ~ \pi \alpha \rho \alpha ́$ de Od. 3.324) en las mismas posiciones. Podría haber otras explicaciones de esto, pero, considerando los resultados de la primera parte de este trabajo, puede entenderse que indica que las paroxítonas y las oxítonas de final resonante tienen una prosodia similar (y ya se ha demostrado -cf. sec. 0.2.2- que las segundas son $\beta \alpha \rho v ́ c$ en última), mientras que las paroxítonas y las oxítonas de final oclusiva tienen un tipo de acento distinto.

\subsubsection{Sílabas finales de yambos}

Nuevamente, se contrastarán en esta sección palabras con final larga natural y palabras con final larga en sandhi. Las tablas 3.3.3.5, 3.3.3.6 y 3.3.3.7 muestran la distribución de los tipos acentuales con los porcentajes por columna para las tesis segunda a sexta del hexámetro, donde puede haber finales yámbicos. Nótese que en la primera la tercera categoría es la de las perispómenas, dado que la vocal larga final permite la aparición de circunflejos, mientras que en las otras dos la tercera categoría es la de las proparoxítonas, dado que en el caso de las palabras con sílaba final cerrada el agudo en antepenúltima en la forma aislada (el tríbraco, u u u) no viola la regla de limitación, y esto se transfiere a la forma en sandhi (donde esa misma marca sí viola la regla).

$\overline{16}$ Dados los comentarios de DAvid (2006: 94-137). 


\begin{tabular}{|c|c|c|c|c|c|c|}
\hline $\begin{array}{l}\text { Fin. Yam. } \\
\text { (V. L.) }\end{array}$ & $2^{\circ}$ pie & $3^{\circ}$ pie & $4^{\circ} \stackrel{p}{p i e}$ & $5^{\circ}$ pie & $6^{\circ}$ pie & Total \\
\hline Oxítonas & $\begin{array}{r}14 \\
(6,45 \%)\end{array}$ & $\begin{array}{r}31 \\
(8,96 \%)\end{array}$ & $\begin{array}{r}8 \\
(2,20 \%)\end{array}$ & $\begin{array}{r}3 \\
(3,26 \%)\end{array}$ & $\begin{array}{r}5 \\
(23,81 \%)\end{array}$ & $\begin{array}{r}61 \\
(5,87 \%)\end{array}$ \\
\hline Paroxítonas & $\begin{array}{r}182 \\
(83,87 \%)\end{array}$ & $\begin{array}{r}275 \\
(79,48 \%)\end{array}$ & $\begin{array}{r}280 \\
(77,13 \%)\end{array}$ & $\begin{array}{r}74 \\
(80,43 \%)\end{array}$ & $\begin{array}{r}13 \\
(61,90 \%)\end{array}$ & $\begin{array}{r}824 \\
(79,31 \%)\end{array}$ \\
\hline Perispómenas & $\begin{array}{r}21 \\
(9,68 \%)\end{array}$ & $\begin{array}{r}40 \\
(11,56 \%)\end{array}$ & $\begin{array}{r}75 \\
(20,66 \%)\end{array}$ & $\begin{array}{r}15 \\
(16,30 \%)\end{array}$ & $\begin{array}{r}3 \\
(14,29 \%)\end{array}$ & $\begin{array}{r}154 \\
(14,82 \%)\end{array}$ \\
\hline Total & 217 & 346 & 363 & 92 & 21 & 885 \\
\hline
\end{tabular}

Tabla 3.3.3.5. Cantidades por ubicación de palabras de final yámbico y última sílaba con vocal larga con diferentes tipos de acento en Homero. Entre paréntesis se agrega el porcentaje por columna.

\begin{tabular}{|l|r|r|r|r|r|r|}
\hline Fin. Yam. (Oc.) & 20 pie & 3ㅇ pie & 4ㅇ pie & 5ㅇ pie & 6ㅇ pie & Total \\
\hline Oxítonas & 1 & 2 & 3 & 0 & 1 & 7 \\
\hline Paroxítonas & $(1,20 \%)$ & $(1,77 \%)$ & $(2,63 \%)$ & $(0 \%)$ & $(3,13 \%)$ & $(1,75 \%)$ \\
\hline Proparoxítonas & 30 & 26 & 71 & 11 & 14 & 152 \\
& $(62,65 \%)$ & $(75,22 \%)$ & $(35,09 \%)$ & $(80,70 \%)$ & $(53,13 \%)$ & $(60,15 \%)$ \\
\hline Total & 83 & 113 & 114 & 57 & 32 & 399 \\
\hline
\end{tabular}

Tabla 3.3.3.6. Cantidades por ubicación de palabras de final yámbico y última sílaba con sílaba cerrada por oclusiva con diferentes tipos de acento en Homero. Entre paréntesis se agrega el porcentaje por columna.

\begin{tabular}{|c|c|c|c|c|c|c|}
\hline Fin. Yam. (Re.) & $2^{\circ}$ pie & $3^{\circ}$ pie & $4^{\circ}$ pie & $5^{\circ}$ pie & $6^{\circ}$ pie & Tota \\
\hline Oxítonas & $\begin{array}{r}5 \\
(9,62 \%)\end{array}$ & $\begin{array}{r}4 \\
(4,76 \%)\end{array}$ & $\begin{array}{r}2 \\
(2,50 \%)\end{array}$ & $\begin{array}{r}3 \\
(7,69 \%)\end{array}$ & $\begin{array}{r}2 \\
(13,33 \%)\end{array}$ & $\begin{array}{r}16 \\
(5,93 \%)\end{array}$ \\
\hline Paroxítonas & $\begin{array}{r}14 \\
(26,92 \%)\end{array}$ & $\begin{array}{r}24 \\
(28,57 \%) \\
\end{array}$ & $\begin{array}{r}53 \\
(66,25 \%) \\
\end{array}$ & $\begin{array}{r}5 \\
(12,82 \%)\end{array}$ & $\begin{array}{r}7 \\
(46,67 \%) \\
\end{array}$ & $\begin{array}{r}103 \\
(38,15 \%)\end{array}$ \\
\hline Proparoxítonas & $\begin{array}{r}33 \\
(63,46 \%)\end{array}$ & $\begin{array}{r}56 \\
(66,67 \%)\end{array}$ & $\begin{array}{r}25 \\
(31,25 \%)\end{array}$ & $\begin{array}{r}31 \\
(79,49 \%)\end{array}$ & $\begin{array}{r}6 \\
(40 \%)\end{array}$ & $\begin{array}{r}151 \\
(55,93 \%)\end{array}$ \\
\hline Total & 52 & 84 & 80 & 39 & 15 & 270 \\
\hline
\end{tabular}

Tabla 3.3.3.7. Cantidades por ubicación de palabras de final yámbico y última sílaba con sílaba cerrada por resonante con diferentes tipos de acento en Homero. Entre paréntesis se agrega el porcentaje por columna. 
Una vez más, en las tablas correspondientes a las sílabas cerradas, el tipo acentual de las formas aisladas tiende a prevalecer por sobre las preferencias de distribución en el metro: las palabras proparoxítonas (como

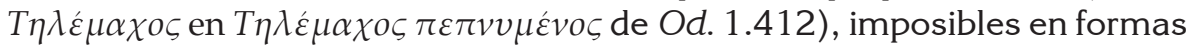
aisladas de final yámbico, prevalecen en casi todas las columnas de las tablas 3.3.3.6 y 3.3.3.7. Incluso en el $4^{\circ}$ pie, donde pareciera que esto se invierte, en realidad la razón por la que hay mayor cantidad de paroxítonas es que hay menor cantidad de formas trisilábicas (dada la incidencia de la cesura trocaica tercera). Sin embargo, las tablas no son inconcluyentes. La distribución en formas pírricas (esto es, de doble breve) de las paroxí-

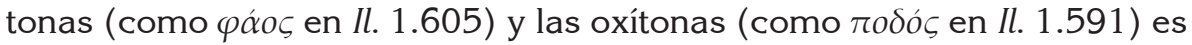
aproximadamente de 2:1 en Homero ${ }^{17}$, pero en la tabla 3.3.3.7 (resonantes) en casi todas las ubicaciones supera el 3:1, y en la 3.3.3.6 (oclusivas) en todas el 10:1. Esto sugiere que existe una tendencia por parte del poeta, cuando utiliza palabras con final breve en su forma aislada en forma yámbica (es decir, $\varphi x ́ o \varsigma$, que es un pírrico, como un yambo, con su sílaba final cerrada), a utilizar aquellas que son paroxítonas por sobre aquellas que son oxítonas. En otras palabras, mientras que debiendo optar entre

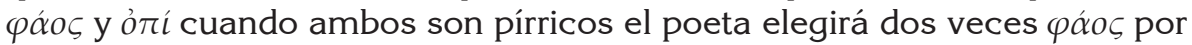
cada vez que elija ỏ $\pi \dot{i}$; debiendo optar entre $\varphi \alpha ́ \alpha \varsigma$ y $\pi o \delta o ́ \varsigma$ cuando ambas tienen sílaba final cerrada por su sibilante optará diez veces por $\varphi$ óos por cada vez que opte por roßós. Esto es compatible con la idea de que cuando sobre estas sílabas cerradas en sandhi cae el acento barítono las palabras son $\beta \alpha \rho v ́$ s sobre la sílaba cerrada.

\subsection{Conclusiones}

Los análisis realizados sugieren que la interpretación más comprehensiva de la prosodia de las sílabas largas con barítono es la correcta, y por lo tanto que en todos los casos éstas deben ser analizadas como $\beta \alpha \rho v ́$ ऽ sobre la sílaba con el barítono. En el estudio de palabras proparoxítonas de final trocaico (anfibráqueos y palimbáqueos) no se hallaron diferencias significativas en la distribución de las diferentes estructuras silábicas, con la excepción de una cierta preferencia por palabras con este tipo acentual en

17 Es decir que hay dos palabras pírricas del tipo acentual de $\varphi \alpha ́ o \varsigma$ por cada palabra pírrica

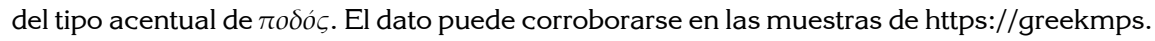
wordpress.com. No lo he incluido especialmente en las tablas correspondientes a este trabajo. La proporción es un poco menor para las palabras con sílaba final abierta (e.g. ỏií en $\partial \pi i k \alpha \lambda \tilde{y}$ en Il. 1.604) y un poco mayor para las palabras con sílaba final con consonante

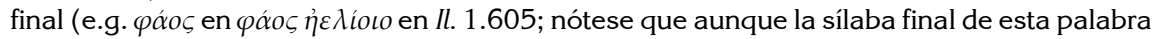
termina con consonante no está cerrada, puesto que en ese caso se volvería un yambo). Varía también de ubicación a ubicación del verso, pero a los fines del presente análisis es la general del texto la que importa. 
el caso de las oclusivas en penúltima. Esta preferencia, de hecho, refuerza la conclusión alcanzada, en la medida en que las palabras con esa estructura no pueden formar $\beta \alpha \rho v ́ \varsigma$ de otra manera que siendo proparoxítonas.

En el análisis de palabras con sílaba acentuada sin modificación en sandhi pero con sílaba final cerrada en el contexto (palabras proparoxítonas de final espondaico), los resultados fueron marcadamente inconcluyentes, dada la escasa cantidad de formas de este tipo. La distribución de estas formas no ofreció motivos para cambiar las conclusiones alcanzadas en el análisis de las anteriores, sin por ello dar argumentos adicionales para sostenerlas.

Finalmente, el estudio de las palabras en las que la sílaba con el barítono cambia su cantidad en sandhi (palabras paroxítonas con final espondaico y con final yámbico), aunque por sí mismo insuficiente para decidir la cuestión, mostró ciertas tendencias compatibles con los resultados de los primeros análisis realizados. En el caso de las palabras con final espondaico se advierte una cierta asociación entre paroxítonas y oxítonas con sílaba final cerrada por resonante y una cierta disociación entre los mismos tipos acentuales en palabras con sílaba final cerrada por oclusiva. Dado que se concluyó en la primera parte de este trabajo que los agudos en estas sílabas cerradas las hacen respectivamente $\beta \alpha \rho \hat{v} \varsigma$ y óćv́, se sugirió que la asociación con las primeras en el caso de las resonantes con barítono y la disociación en el caso de las oclusivas con barítono indica que ambas hacen $\beta \alpha \rho v ́ c$ a la palabra. El estudio de las palabras yámbicas mostró resultados similares: la preferencia por formas paroxítonas por sobre oxítonas (en razones superiores en general a 3:1 y en las oclusivas a 10:1) en palabras que en su forma aislada son pírricos fue tomada como indicio de un cambio de prosodia, dado que la proporción entre esos tipos acentuales es en Homero de 2:1 en los pírricos cuando aparecen como pírricos.

Esta segunda parte del trabajo no obtuvo resultados tan contundentes y claros como la primera, pero la evidencia permite afirmar, como conclusión general, que todas las palabras con barítono sobre sílaba larga, independientemente de su estructura acentual, eran consideradas $\beta \alpha \rho v ́ \varsigma$ sobre esa sílaba larga. Es probable que hubiera distinciones sutiles, y éstas en algunos casos pueden inferirse a partir de los datos expuestos; sin embargo, se requiere un estudio más localizado de cada una de las formas para detectar estas distinciones. A los fines del presente estudio, el problema de los barítonos sobre sílaba larga puede considerarse resuelto. 


\section{Una reflexión final sobre la naturaleza de la evidencia}

Me gustaría cerrar este trabajo haciendo notar que la premisa metodológica fundamental para que fuera desarrollado, esto es, que los poemas homéricos (junto con otras formas de poesía que aquí no han sido utilizadas) son de hecho fuente admisible para el estudio del acento griego, no es una idea generalizada en la disciplina ni, probablemente, aceptada sin más. Mientras que, por un lado, esto puede razonablemente arrojar una sombra de duda sobre las conclusiones alcanzadas, no deja de ser cierto también que la evidencia obtenida deja traslucir una y otra vez decisiones del poeta que parecen ser atribuibles a la preferencia o la evasión de ciertos tipos acentuales. Existe, sin duda, la posibilidad (cf. nn. 39 y 40 de la primera parte) de buscar otras explicaciones para estas preferencias, ya sean métricas o simplemente lexicales (la disponibilidad de ciertos tipos acentuales en ciertas formas, por ejemplo), y es importante que, por improbables que estos caminos de investigación resulten, sean al menos tomados en cuenta. No es recomendable que la tentación de abrir un nuevo campo de exploración o de recuperar la melodía de la poesía antigua derive en una aceptación irreflexiva de la premisa de que el acento cumple un rol importante en ella. Pero, nuevamente, los resultados que se han obtenido sugieren fuertemente que este camino se ha abierto. La circularidad del razonamiento no se me escapa, pero es falaz: el hecho de que el axioma metodológico fundamental de este trabajo haya sido verificado con la evidencia simplemente demuestra que es un axioma metodológico adecuado. El razonamiento es abductivo, pero suelen serlo los que permiten realizar avances en la ciencia. En cualquier caso, si algo debe resultar evidente a partir de los análisis realizados en este trabajo, que sea que la premisa de que la poesía griega no era indiferente a los acentos del lenguaje no puede ser inmediatamente descartada. 


\section{Bibliografía $^{18}$}

Allen, W. S. (1987) Vox Graeca, Cambridge, University Press. (1973) Accent and Rhythm, Cambridge, University Press.

David, A. P. (2006) The Dance of the Muses. Choral Theory and Ancient Greek Poetics, Oxford: University Press.

Devine, A.M. y Stephens, L. D. (1994) The Prosody of Greek Speech. New York and Oxford, Oxford University Press.

Nagy, G. (1996) Poetry as Performance: Homer and Beyond, Cambridge, Cambridge University Press.

(2000) "Reading Greek Poetry Aloud. Evidence from the Bacchylides Papyri”, QUCC 64, pp. 7-28.

(2010) "Language and Meter", en E. J. Bakker (ed.) A Companion to the Ancient Greek Language, West Sussex: Wiley-Blackwell, pp. 370-387.

Cantinela, M. (1995), "Il ponte di Nicanore", en M. Fantuzzi, R. Pretagostini (eds.) Struttura e storia dell'esametro greco, vol. I, Roma, Gruppo Editoriale Internazionale, pp. 9-68.

Fecha de recepción: 25-06-2015

Fecha de aceptación: 17-12-2015

18 Una bibliografía más extensa sobre los problemas analizados puede hallarse en la primera parte de este estudio, publicado en el Volumen 39 de esta revista. 